\title{
Output-Only Modal Analysis of Linear Time Periodic Systems with Application to Wind Turbine Simulation Data
}

\author{
Matthew S. Allen, \\ Assistant Professor \\ msallen@engr.wisc.edu \\ Michael W. Sracic \\ Graduate Research Assistant, Ph.D. Candidate \\ mwsracic@wisc.edu \\ Department of Engineering Physics \\ University of Wisconsin-Madison \\ 535 Engineering Research Building \\ 1500 Engineering Drive \\ Madison, WI 53706 \\ Shashank Chauhan \\ Brüel \& Kjær \\ Sound \& Vibration Measurement A/S \\ Skodsborgvej 307 \\ DK-2850 Nærum, Denmark \\ schauhan@bksv.com
}

$\&$

Morten Hartvig Hansen

Wind Energy Department

Risø National Laboratory

P.O. Box 49, DK-4000 Roskilde

Denmark

\begin{abstract}
:
Many important systems, such as turbomachinery, helicopters and wind turbines, must be modeled with linear time-periodic equations of motion to correctly predict resonance phenomena. Time periodic effects in wind turbines might arise due to stratification in the velocity of the wind with height, changes in the aerodynamics of the blades as they pass the tower and/or blade-to-blade manufacturing variations. These effects may cause parametric resonance or other unexpected resonances, so it is important to properly characterize them so that these machines can be designed that achieve the high reliability, safety, and long lifetimes demanded to provide economical power. This work presents an output-only system identification methodology that can be used to identify models for linear, periodically time-varying systems. The methodology is demonstrated for the simple Mathieu oscillator and then used to interrogate simulated measurements from a rotating wind turbine, revealing that at least one of the turbine's modes seems to be time-periodic. The measurements were simulated using a state-of-the-art wind turbine model called HAWC2 that includes both structural dynamic and aerodynamic effects. Simulated experiments such as this may be useful both to validate dynamic models for a turbine or to obtain a set of time-periodic equations of motion from a numerical model; these are not readily available by other means due to the way that the aeroelastic effects are treated in the simulation code.
\end{abstract}




\section{Introduction}

Linear time-periodic (LTP) differential equations are important for quite a wide range of systems including helicopters [1-7], turbomachinery [8-10] wind turbines [11-13] and virtually any system that can be linearized about a periodic trajectory (see, e.g. [14-17]). Allen \& Sracic have even proposed that a general nonlinear system might be more easily identified if excited such that an LTP model is appropriate [18]. Other researchers have sought to exploit LTP effects to detect defects, such as a cracked shaft, in a rotating system [8-10].

This work explores LTP identification for wind turbines. Time-periodic models are necessary to model wind turbine dynamics if the turbine has only two blades, or if the blades of a 3+ blade turbine are not perfectly identical, since the turbine tower is always anisotropic. In practice there are always differences between the blades but these are often not considered in design, analysis or even when performing tests. Time-periodicity can also arise due to aeroelastic effects such as interaction between the flow around the blade and tower or variation in the wind speed with altitude. Most of these effects are difficult to model and there is considerable uncertainty in the parameters that should be used, so experimental methods are needed both to validate existing models and to probe experimental measurements to see whether time-periodic effects should be included in future models.

A few system identification strategies have been proposed for linear time-periodic systems. Many of them are extensions of system realization methods (e.g. the Eigensystem Realization Algorithm [19].) For example, Liu presented a discrete-time approach of this form in [20], Verhagen presented a subspace method [21], and others have investigated this as well $[22,23]$. Peters and Fuehne independently developed a related approach that they call Generalized Floquet Theory [2, 7, 24, 25], which seeks to derive the Floquet exponents of the time-periodic system from measured time series. Other researchers have explored optimization-based approaches [26, 27]. While those works are valuable, frequency domain system identification approaches are preferred in many instances because one can more easily make sense of complicated measurements and averaging can be performed to reduce long, cumbersome time series to a compact representation of the system's response. Wereley and Hall extended the concept of the frequency response function (FRF) to LTP systems [28-30], paving the way for frequency domain identification of LTP systems. A number of other researchers subsequently presented many similar concepts [31], one group taking a complex frequency response approach [32]. However, to date only a few works have sought to implement these concepts experimentally and sometimes the results have been disappointing [3,5,33]. One exception is continuous-scan laser Doppler vibrometry (CSLDV), which can also be thought of as a special case of LTP system identification. A number of effective methods have been developed for CSLDV and they have been used with real measurements demonstrating very good results in many cases [34-42]. On the other hand, many of those methods are specialized to CSLDV and not immediately applicable to time-periodic systems in general.

This work uses the harmonic transfer function concept developed by Wereley [28] to create an outputonly system identification method for linear time-periodic systems. The proposed method can be thought of as an extension of Operational Modal Analysis (OMA) [43-45] or Output-only modal analysis [46-48] to time-periodic systems. One of the first OMA algorithms in the structural dynamics community was Natural Excitation Technique (NExT) [49] by James \& Carne, and in the intervening years there have been hundreds of papers on OMA and there is even now a conference devoted to the topic. As with OMA for time-invariant systems, the theory presented here can be used to extract the time-periodic mode vectors of a system from an (augmented) auto-spectrum of the response if the input obeys certain assumptions.

The rest of this paper is organized as follows. Section 2 derives the proposed output-only identification technique with particular emphasis on how to interpret the response spectra of an LTP system. In Section 3 the proposed methodology is demonstrated by identifying a model for a simple time-periodic system (the Mathieu oscillator) from simulated measurements. The method is then applied to simulated measurements from a wind turbine in Section 4, and Section 5 summarizes the conclusions.

\section{Theoretical Development}

The state space equations of motion of a linear time-periodic (LTP) system can be written as follows,

$$
\dot{x}=A(t) x+B(t) u \quad, \quad y=C(t) x+D(t) u
$$


where $A\left(t+T_{A}\right)=A(t)$ and the other matrices are periodic as well with the same period. The fundamental frequency of the time-periodic system is denoted $\omega_{A}=2 \pi / T_{A}$. The state transition matrix gives the free response of such a system at time $t$ via the relationship,

$$
x(t)=\Phi\left(t, t_{0}\right) x\left(t_{0}\right) .
$$

One can also write the forced response of the system in terms of the STM as follows.

$$
y(t)=C(t) \Phi\left(t, t_{0}\right) x\left(t_{0}\right)+\int_{t_{0}}^{t} C(t) \Phi(t, \tau) B(\tau) u(\tau) d \tau
$$

Floquet theory reveals that, in the absence of degenerate roots, the state transition matrix of an LTP system can be represented as a modal sum $[9,10]$,

$$
\Phi\left(t, t_{0}\right)=\sum_{r=1}^{N} \psi_{r}(t) L_{r}\left(t_{0}\right)^{T} \exp \left(\lambda_{r}\left(t-t_{0}\right)\right)
$$

where $\lambda_{r}$ is the $r$ th Floquet exponent of the state transition matrix, $\psi_{r}$ is the $r$ th time-periodic mode vector of the STM and $L_{r}$ is the $r$ th column of $\left[\begin{array}{llll}\psi_{1}(t) & \psi_{2}(t) & \cdots\end{array}\right]^{-T}$. The Floquet exponents of an LTP system are analogous to the eigenvalues of a Linear Time Invariant (LTI) system, which can be written in terms of the damping ratio $\zeta_{r}$ and natural frequency $\omega_{r}$ as $\lambda_{r}=-\zeta_{r} \omega_{r}+i \omega_{r} \sqrt{1-\zeta_{r}{ }^{2}}$ for an underdamped mode. (Note that the eigenvectors of $A(t)$ have not been mentioned here; the modal parameters of the STM are more useful for determining the stability of the system and understanding its response, and they are more convenient to identify experimentally, so we shall focus on them. For an LTI system the STM and $A(t)$ share the same eigenvectors. Most system identification routines for LTI systems also seek to find the modal parameters of the STM, for example those based on a discrete-time representation, which is a special case of eq. (3) for a constant time step (see, e.g. [45, 50].)

Since the eigenvectors of the STM are periodic, it is convenient to expand them in a Fourier series. In practice the state vector is usually not measured but some vector of outputs $(y(t)$ in eq. (1)), so we shall concern ourselves only with the observed mode vectors, $C(t) \psi_{r}(t)$. As is often the case for LTI systems, $C(t)$ may simply be a matrix of ones and zeros indicating which of the states were measured, or one may have more measurement points than states and then $C(t)$ has more rows than columns. In any event the expansion is,

$$
C(t) \psi_{r}(t)=\sum_{n=-\infty}^{\infty} \bar{C}_{r, n} e^{j n \omega_{A} t} \quad, \quad L_{r}(t)^{T} B(t)=\sum_{n=-\infty}^{\infty} \bar{B}_{r, n} e^{j n \omega_{A} t}
$$

where $\bar{C}_{r, n}$ is the $n$th vector of Fourier coefficients of the $r$ th observed mode vector, $C(t) \psi_{r}(t)$, and similarly for $L_{r}(t)^{\mathrm{T}} B(t)$.

It is well known that the response of a LTI system to a complex exponential input, $u(t)$, results in a complex exponential output, $y(t)$, although with different magnitude and phase. In contrast, a linear time-periodic system may respond to a single sinusoid at an infinite number of frequencies, which caused some to believe that the concept of a transfer function couldn't be extended to LTP systems [31]. Wereley addressed this difficulty using an exponentially modulated periodic (EMP) signal space, in which $u$ and $y$ are described as follows.

$$
u(t)=\sum_{n=-\infty}^{\infty} u_{n} e^{\left(j \omega+j n \omega_{A}\right) t} \quad, \quad y(t)=\sum_{n=-\infty}^{\infty} y_{n} e^{\left(j \omega+j n \omega_{A}\right) t}
$$

These signals are useful because an EMP input signal causes an EMP output, although with different magnitude and phase at each frequency in the comb, so a concept that is completely analogous to the LTI transfer function can be derived. This is done by defining vectors of the harmonics, $y_{n}$, of output, $\mathbf{y}(\omega)=\left[\begin{array}{lllll}\cdots & y_{-1}{ }^{T} & y_{0}{ }^{T} & y_{1}{ }^{T} & \cdots\end{array}\right]^{T}$, and similarly for the input, then writing a transfer function relating input and output EMP signals, which is dubbed the Harmonic Transfer Function (HTF) [28]. 


$$
\mathbf{y}(\omega)=\mathbf{G}(\omega) \mathbf{u}(\omega)
$$

One can also write $\mathbf{y}$ as,

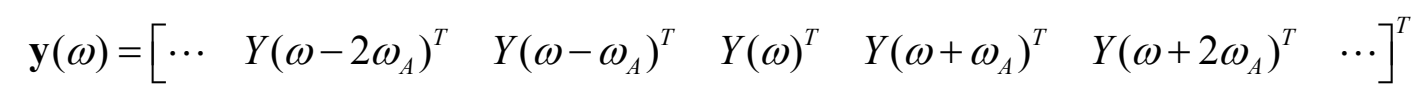

where $Y(\omega)$ is the Fourier transform of $y(t)$. Hence, one can construct the EMP signals $\mathbf{u}$ and $\mathbf{y}$ from measurements of the input and response $u(t)$ and $y(t)$. Theoretically, one must consider an infinite number of harmonics to characterize an LTP system, yet one would expect that most systems can be well approximated with a finite, perhaps even small number.

For LTI systems, the FRF matrix has a well-known form with peaks near the natural frequencies of each mode, and with the FRF matrix approximating the shape of the corresponding eigenvector near that frequency. A similar relationship must be derived for LTP systems. This was done by substituting eq. (4) into eq. (3), dropping transient terms (assuming steady state) and using a Fourier series expansion to simplify the expression. Wereley's thesis shows an example of this type of calculation. After much algebra, one obtains the following expression for $\mathbf{G}$ in terms of the modal parameters of the STM.

$$
\begin{aligned}
& \mathbf{G}(\omega)=\sum_{r=1}^{N} \sum_{l=-\infty}^{\infty} \frac{\overline{\mathbf{C}}_{r, l} \overline{\mathbf{B}}_{r, l}}{i \omega-\left(\lambda_{r}-i l \omega_{A}\right)}+\mathbf{D} \\
& \overline{\mathbf{C}}_{r, l}=\left[\begin{array}{llllll}
\cdots & \bar{C}_{r,-1-l}{ }^{T} & \bar{C}_{r,-l}^{T} & \bar{C}_{r, 1-l}{ }^{T} & \cdots
\end{array}\right]^{T} \\
& \overline{\mathbf{B}}_{r, l}=\left[\begin{array}{llllll}
\cdots & \bar{B}_{r, l+1} & \bar{B}_{r, l} & \bar{B}_{r, l-1} & \cdots
\end{array}\right]
\end{aligned}
$$

where the $n$th term in the vector $\overline{\mathbf{C}}_{r, l}$, is $\bar{C}_{r, n-l}$, the $(n-l)$ th Fourier coefficient of $C(t) \psi_{r}(t), \bar{B}_{r, l-m}$ is the $(l-m)$ th Fourier coefficient of $L_{r}(t)^{\mathrm{T}} B(t)$ and the $(n, m)$ th element of $\mathbf{D}$ is the $(n-m)$ th Fourier coefficient of $D(t)$. This expression has exactly the same mathematical form as the expression for the FRF matrix in terms of the modal parameters for a linear time-invariant system, so the same algorithms can be applied to identify the parameters of the LTP system and the same intuition that one uses to interpret FRFs can also be used to interpret HTFs. There are, however, a few differences that must be noted:

- An LTP system of order $N$ can potentially have an infinite number of peaks in its HTF, depending on how many terms in $\overline{\mathbf{C}}_{r, l}$ and $\overline{\mathbf{B}}_{r, l}$ are nonzero. Each peak will occur near the imaginary part of the Floquet exponent $\lambda_{r}$ plus some integer multiple of the fundamental frequency $\omega_{A}$. If the mode shapes of the system are constant in time, then $\overline{\mathbf{C}}_{r, l}$ and $\overline{\mathbf{B}}_{r, l}$ contain only one nonzero term and eq. (9) reduces to the familiar relationship for an LTI system.

- Whereas the mode shapes of an LTI system describe the spatial pattern of deformation of a mode, the observed mode shapes of an LTP system, $\overline{\mathbf{C}}_{r, l}$, also reveal how that shape changes with time. A singleoutput LTP system still has a mode shape that is a vector, with each element in that vector being a Fourier coefficient describing how the mode shape at that one output point changes with time.

In the applications that are of interest in this work the input is not known, so we desire to determine the modal parameters of the system from the output signal only. To do this, we write the power spectrum of the output in terms of the HTF matrix and the input, resulting in the following,

$$
S_{y y}(\omega)=\mathbf{G}(\omega) S_{u u}(\omega) \mathbf{G}(\omega)^{H}
$$

where the power spectra can be found by applying the usual estimation algorithm to the EMP signals,

$$
S_{y y}(\omega)=\frac{1}{M} \sum_{k=1}^{M} \mathbf{y}_{k}(\omega) \mathbf{y}_{k}(\omega)^{H}
$$

with ()$^{H}$ denoting the Hermitian or complex-conjugate transpose. Replacing $\boldsymbol{G}$ with its modal representation in eq. (10), one obtains the following for the case where $D(t)=0$, 


$$
\begin{gathered}
{\left[S_{y y}(\omega)\right]=\sum_{r=1}^{N} \sum_{l=-\infty}^{\infty} \sum_{s=1}^{N} \sum_{k=-\infty}^{\infty} \frac{\overline{\mathbf{C}}_{r, l} \mathbf{W}(\omega)_{r, s, l, k} \overline{\mathbf{C}}_{s, k}{ }^{H}}{\left[i \omega-\left(\lambda_{r}-i l \omega_{A}\right)\right]\left[i \omega-\left(\lambda_{s}-i k \omega_{A}\right)\right]^{H}}} \\
\mathbf{W}(\omega)_{r, s, l, k}=\overline{\mathbf{B}}_{r, l} S_{u u}(\omega) \overline{\mathbf{B}}_{s, k}{ }^{H}
\end{gathered}
$$

where $\mathbf{W}(\omega)$ is a function of the input spectrum and the input characteristics of the system. This shows that the autospectrum of the output can be approximated by a sum of modal contributions if $\mathbf{W}(\omega)$ is reasonably flat. (For LTI systems this requires that the input spectrum $S_{u u}(\omega)$ be flat. Here the requirement is similar, yet perhaps more stringent.) The dominant terms in the summation above are those for which $j \omega-\left(\lambda_{r}-j l \omega_{A}\right)$ and $j \omega-\left(\lambda_{s}-j k \omega_{A}\right)$ are both minimum at the same frequency. If the sidebands for mode $r$ do not overlap with those for mode $s$, then this occurs when $r=s$ and $l=k$ and the expression becomes,

$$
\left[S_{y y}(\omega)\right]=\sum_{r=1}^{N} \sum_{l=-\infty}^{\infty} \frac{\overline{\mathbf{C}}_{r, l} \mathbf{W}(\omega)_{r, l} \overline{\mathbf{C}}_{r, l}{ }^{H}}{\left[i \omega-\left(\lambda_{r}-i l \omega_{A}\right)\right]\left[i \omega-\left(\lambda_{r}-i l \omega_{A}\right)\right]^{H}}
$$

so the shape of the autospectrum is proportional to the dominant mode shape and a particular element of the autospectrum plotted versus frequency has the shape of an LTI mode squared. The extension of these ideas to nonzero $D(t)$ is straightforward. As with operational modal analysis for LTI systems, there are additional scale factors that must be determined to find the response of the LTP system to an input or an initial condition, but the modal parameters are still useful for evaluating the response of the system qualitatively.

The proposed output-only system identification routine can be summarized as follows:

1. Record the response, $y(t)$, of an LTP system to a broadband input.

2. Form a vector, $\mathbf{y}$, of frequency shifted copies of the output as shown in eq. (8) by using the fact that $Y(\omega-$ $\left.n \omega_{A}\right)=\mathrm{FFT}\left[y(t) * \exp \left(-i n \omega_{A} t\right)\right]$.

3. Compute the autospectrum of $\mathbf{y}$ by averaging multiple blocks of the time history as shown in eq. (11).

4. Use an LTI output only system identification routine to identify the modal parameters from the autospectrum, using eqs. (12) and (13) to interpret the results and reconstruct the modal parameters of the STM. One of the simplest applicable identification methods is the peak picking method.

5. Reconstruct $C(t) \psi_{r}(t)$, using the identified Fourier coefficients, $\overline{\mathbf{C}}_{r, l}$, if desired.

\section{Simulated Application: Mathieu Oscillator}

A single degree-of-freedom Mathieu Oscillator was used to evaluate the proposed identification methodology on a very simple time-periodic system. Figure 1 shows a schematic of the Mathieu Oscillator.

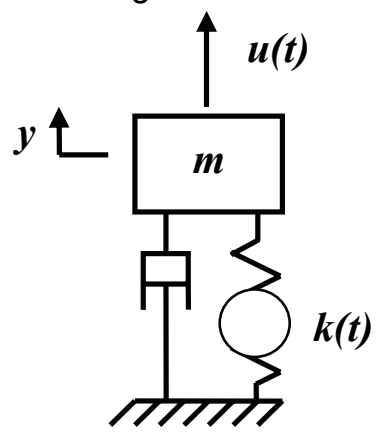

Figure 1: Mathieu Oscillator with a time varying spring stiffness.

The spring stiffness for the system varies periodically with frequency $\omega_{A}$ according to eq. (14).

$$
k(t)=k_{0}+k_{1} \cos \left(\omega_{A} t\right)
$$

After normalizing by dividing out the oscillator mass, the system has the following equation of motion 


$$
\ddot{y}+2 \zeta \omega_{0} \dot{y}+\left(\omega_{0}^{2}+\omega_{1}^{2} \cos \left(\omega_{A} t\right)\right) y=\frac{u(t)}{m}
$$

with $2 \zeta \omega_{0}=c / m, \omega_{0}^{2}=k_{0} / m, \omega_{1}^{2}=k_{1} / m$, and input $u(t)$.

The Mathieu oscillator was used to simulate an output-only response. The parameters used for the oscillator were $m=1, k_{0}=1, k_{1}=0.4$, and $\omega_{A}=0.8 \mathrm{rad} / \mathrm{s}$. A damping ratio of 0.02 was desired so the damping coefficient was set to $c=0.4$. The system was subjected to wide-band random white noise input, which was generated by passing a vector of randomly generated numbers through an $8^{\text {th }}$-order low-pass Butterworth filter with a cutoff frequency of $10 \mathrm{rad} / \mathrm{s}$. Figure 2 shows a plot of the input signal.

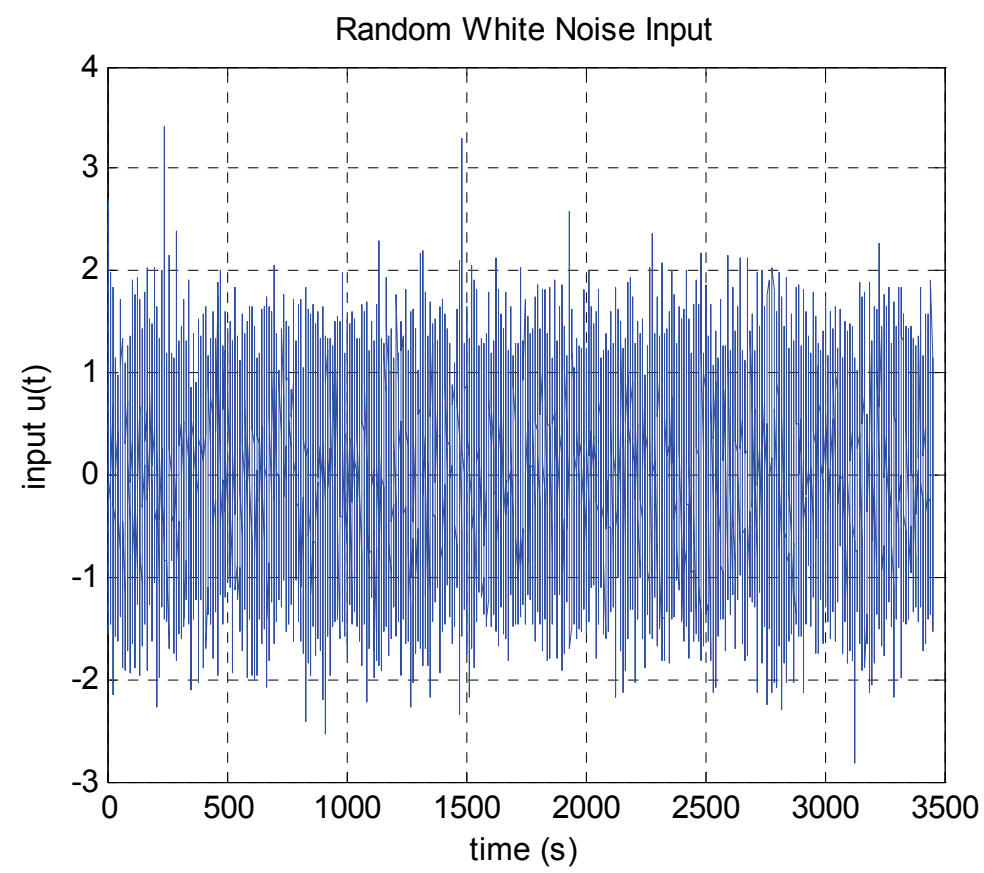

Figure 2: Random white noise input signal.

The response of the Mathieu oscillator was then determined by integrating the system's state space equations from zero initial conditions with MATLAB's adaptive Runge-Kutta (ode45) routine over the duration of the white noise input signal. The input was then discarded and the output of the integration treated as a measurement. The output measurement was then exponentially modulated with harmonics $n=-2,-1,0,1$, and 2 to form $y$ in eq. (8), the EMP output signal. The output power spectrum was then calculated according to eq. (11). Figure 3 shows a plot of all of the terms in the power spectrum matrix along the primary row (e.g. with $m=0$ and $n$ $=-2 \ldots 2)$. The legend gives the $n$-value for each term. Each of the spectra show strong peaks near $\omega=1 \mathrm{rad} / \mathrm{s}$, which would be the natural frequency of the Mathieu oscillator in the case where $k_{1}=0$ (e.g. a time-invariant system). The $n=0$ spectrum contains the largest peak and the remaining become progressively smaller for $n=-$ $1,1,-2$, and then 2. Two other strong groups of peaks appear in all of the spectra at $\omega=0.2 \mathrm{rad} / \mathrm{s}$, where the peaks have the following order of dominance, $n=[1,0,-1,2,-2]$ and $\omega=1.8 \mathrm{rad} / \mathrm{s}$ with ordering $n=[-1,-2,0,1,2]$. The remaining groupings of peaks occur at $\omega=2.6 \mathrm{rad} / \mathrm{s}$ and $\omega=0.6 \mathrm{rad} / \mathrm{s}$, and there is evidence of a peak in the $n=-2$ curve near $\omega=3.4 \mathrm{rad} / \mathrm{s}$. 


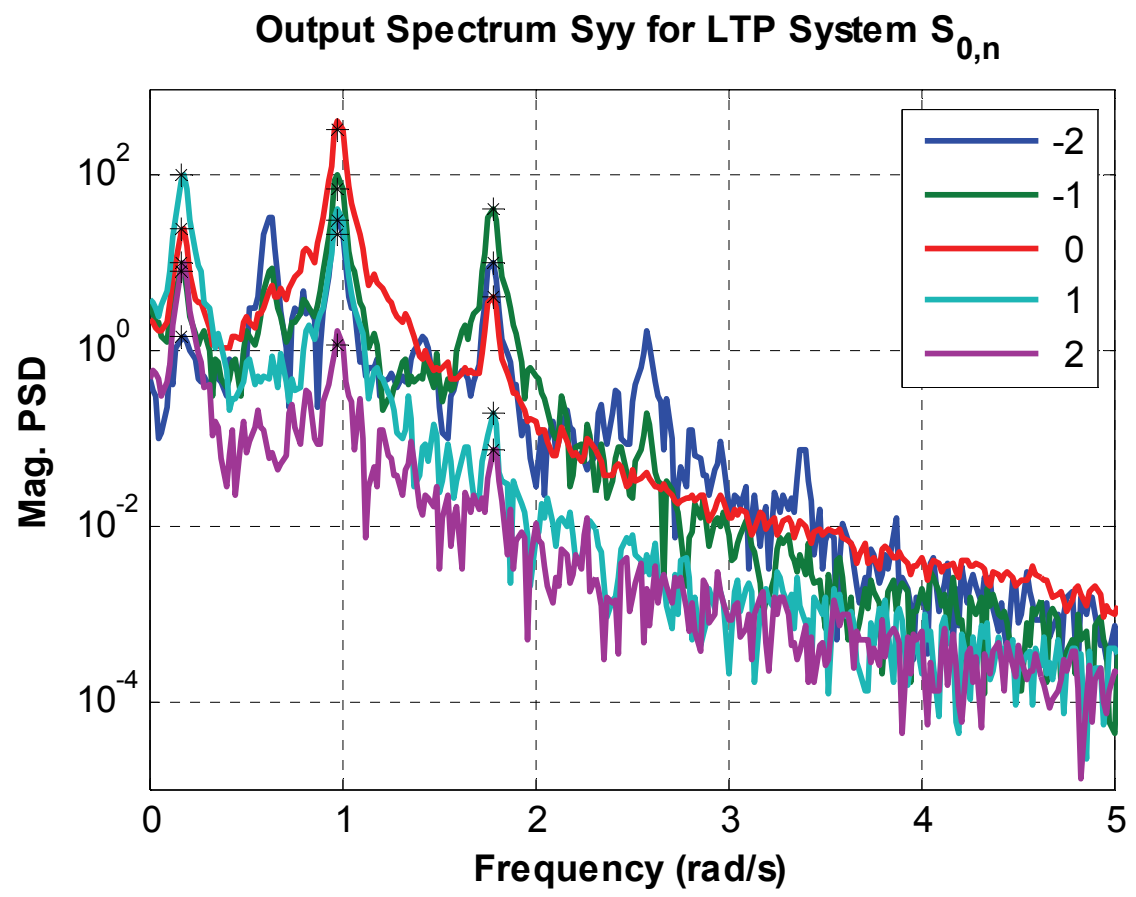

Figure 3: Harmonic PSD of the output for the Mathieu oscillator response computed using eqs. (8) and (11).

Five curves are shown corresponding to the $m$, nth element of $S_{y y}$ for $m=0$ and $n=-2 \ldots 2$.

The peak picking method was used to find the shape of the power spectrum matrix at the peaks at $\omega=0.2$, 1 , and $1.8 \mathrm{rad} / \mathrm{s}$. The values of the spectra from each of those peaks were normalized by dividing by the largest value so that the shape could be compared, and the result is shown in Table (1). The analytical Fourier Coefficients for the Mathieu oscillator were also found by integrating the LTP equations of motion over the fundamental period, solving for $\psi(t)$ and then expanding it in a Fourier series to obtain the vector $\overline{\mathbf{C}}_{r, l}$ in eq. (9). Those Fourier coefficients were then normalized by the maximum and are also shown. (Additional detail regarding how $\overline{\mathbf{C}}_{r, l}$ was derived is provided in Appendix A.)

\begin{tabular}{|c|c|c|c|c|}
\hline \multirow{2}{*}{$n$} & \multicolumn{4}{|c|}{ Fourier Coefficients of the Mathieu Oscillator Mode Shape: } \\
\hline & Analytical & $0.2 \mathrm{rad} / \mathrm{s}$ & $1 \mathrm{rad} / \mathrm{s}$ & $1.8 \mathrm{rad} / \mathrm{s}$ \\
\hline-3 & 0.0135 & $0.0047+0.014 \mathrm{i}$ & - & - \\
\hline-2 & 0.067 & $0.076+0.024 \mathrm{i}$ & $0.064+0.015 i$ & - \\
\hline-1 & -0.220 & $-0.26+0.0009 i$ & $-0.21-0.0021 i$ & $-0.24+0.0011 i$ \\
\hline $\mathbf{0}$ & 1 & 1 & 1 & 1 \\
\hline 1 & 0.092 & $0.097+0.0065 i$ & $0.095+0.0043 i$ & $0.10+0.0068 i$ \\
\hline 2 & 0.0032 & - & $0.0036+0.0006 i$ & $0.0044+0.0009 i$ \\
\hline 3 & 0.00004 & - & - & $-0.0011-0.0014 i$ \\
\hline
\end{tabular}

Table 1 Identified mode shape coefficients at peaks occurring at $\omega=0.2,1.0$ and $1.8 \mathrm{rad} / \mathrm{s}$ for spectra corresponding to $n=-3,-2,-1,0,1,2$ and 3. The analytical Fourier coefficients of the Floquet mode shape of the Mathieu oscillator are also provided.

Equations (12) and (13) revealed that each mode of an LTP system is manifest at multiple frequencies in the output PSD (i.e. for various $l$ ). If the linear term in the mode shape is the largest, then one can surmise that the peak occurring in the primary $(0,0)$ element of the PSD matrix will be at the true natural frequency.

(Mathematically, it does not matter which peak is the primary, so this assumption can always be made and one will still obtain a valid model.) Following this line of thinking, the dominant peak in Figure (3) occurs at $\omega=1.0$ 
$\mathrm{rad} / \mathrm{s}$, so the Floquet exponent is taken to be $\lambda=1.0 i$, and the corresponding natural frequency is $\omega_{0}=1.0$. The remaining peaks in the spectra can be attributed to modulations of this Floquet exponent by the system's fundamental frequency $\omega_{A}=0.8 \mathrm{rad} / \mathrm{s}$. For example, the peaks $0.2 \mathrm{rad} / \mathrm{s}$ can be seen as $\omega_{0}-1 * \omega_{A}$ while the peaks at $1.8 \mathrm{rad} / \mathrm{s}$ can be seen as $\omega_{0}+1 * \omega_{A}$. The peaks at $0.6 \mathrm{rad} / \mathrm{s}$ are due to the second harmonic of the conjugate of the Floquet exponent at $-1.0 \mathrm{rad} / \mathrm{s}$ (i.e. $0.6 \mathrm{rad} / \mathrm{s}=-\omega_{0}+2 * \omega_{A}$ ). Using this reasoning, the Fourier coefficients can be arranged in rows as shown in Table 1. There are multiple estimates for the Fourier coefficients for $n=-1 \ldots 1$, and all of the estimates agree quite well suggesting that the identification was successful. Other coefficients were only estimated from a few of the frequencies (columns), but those are small so it appears that the Fourier series expansion is converging. Comparing the Fourier coefficients identified by the output only technique with the analytical ones, one observes that the agreement is quite good. One could now average the Fourier coefficients that were obtained, reconstruct $C(t) \psi(t)$ and diagnose troublesome frequencies in the response. All of the above was surmised from the output response and without any knowledge of the system except the fact that it was timeperiodic with period $\omega_{A}$. If the scale factors on the mode shape $C(t) \psi(t)$ could be estimated by some means, then one could also reconstruct the time-varying state matrix of the system using the procedure in [10].

\section{Simulated Application: Wind Turbine modeled with HAWC2}

The proposed methodology was also applied to simulated measurements from a representative 5MW wind turbine (3-blade, 126 meter diameter, 100 meter tower height) [51] modeled using the HAWC2 simulation tool [52-54]. This same turbine and simulation code were used in a previous paper where Subspace Identification and Blind Source Separation were used to identify the modes of the turbine when it was in a parked condition [55]. That work identified the lower modes of the turbine, which had the following frequencies: $0.273 \mathrm{~Hz}$ (lateral tower bending), $0.275 \mathrm{~Hz}$ (longitudinal tower bending), $0.564 \mathrm{~Hz}$ (drive train torsion), $0.604 \mathrm{~Hz}$ (yaw), $0.635 \mathrm{~Hz}$ (tilt), $0.698 \mathrm{~Hz}$ (symmetric flapwise bending), $0.951 \mathrm{~Hz}$ (edgewise vertical bending), $0.975 \mathrm{~Hz}$ (edgewise horizontal bending), $1.526 \mathrm{~Hz}, 1.655 \mathrm{~Hz}, 1.74 \mathrm{~Hz}$. Representative mode shapes are also shown in [55]. Here the rotating turbine will be studied. The HAWC2 model includes a structural model based on Timoshenko beam elements for the bending of the blades and tower, aerodynamic excitation of the wind turbine from turbulence in the flow, dynamic inflow, dynamic stall, skew inflow, shear effects on the induction and effects from large deflections. The wind speed for this model is $18 \mathrm{~m} / \mathrm{s}$ at hub height with a logarithmic wind shear. Because of the wind shear, the flow around each blade changes as the blade rotates, so one would expect that the system might exhibit timeperiodic dynamics due to the periodically changing aeroelasticity of the blades.

Measurements were simulated from five points along each blade and ten points along the tower, in all three directions at each point for a total of 75 measurement locations. The rotational speed of the turbine and its instantaneous angle were also "measured", the former averaging $0.20157 \mathrm{~Hz}$ (12.1 rev. per minute) with a standard deviation of $0.000312 \mathrm{~Hz}$. The traditional power spectrum of the response was found using eight sensors as references: the lateral and longitudinal sensors at the tip of the tower and the edgewise and flapwise sensors at the tip of each blade, so the resulting PSD matrix was 75 by 8 . Figure 4 shows the average of all of these power spectra, as well as the average of only those elements of the PSD matrix corresponding to the blades and the tower individually. 


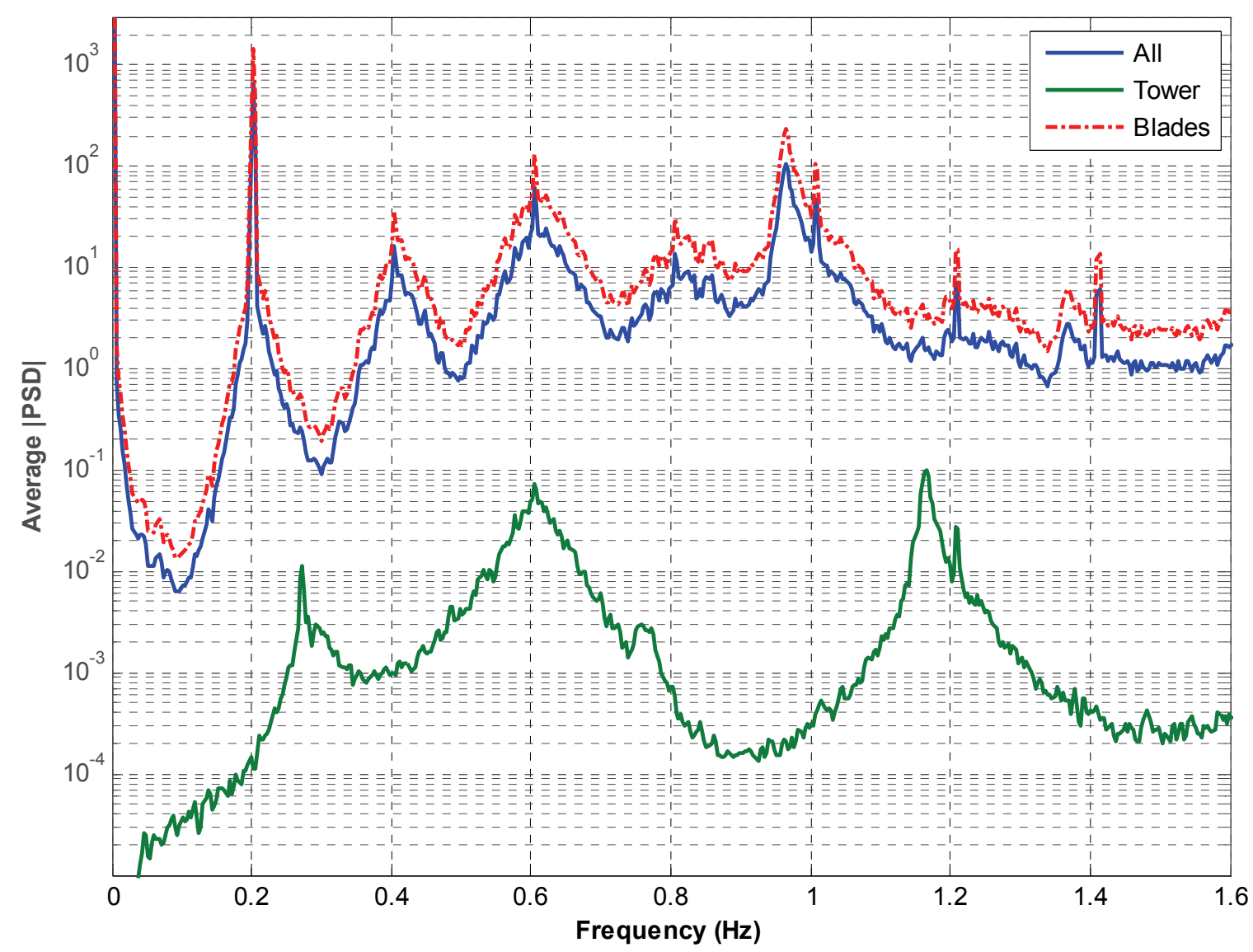

Figure 4: Average of Traditional Power Spectra of rotating wind turbine over all measurement points on the tower and over all of the blades. The average rotational speed of the turbine is $0.20157 \mathrm{~Hz}$.

The effect of the rotation of the blades is readily apparent in the PSD, especially if one compare Figure 4 with the power spectra shown in [55] for the parked condition. There is a strong harmonic at the rotation frequency $0.2 \mathrm{~Hz}$, and other significant ones at integer multiples of the rotation frequency. There are also broad, triangular-shaped peaks in the blade spectra at $0.2,0.4$ and $0.6 \mathrm{~Hz}$, as well as a weaker one at $0.8 \mathrm{~Hz}$. In the tower spectra there is only one such peak at $0.6 \mathrm{~Hz}$, which is the frequency with which blades pass the tower. These peaks dominate the spectra at those frequencies, so none of the modes of the turbine are visible except for the peaks near 0.27 and $1.15 \mathrm{~Hz}$ in the tower response and the one near $0.95 \mathrm{~Hz}$ in the blade response.

Comparing these frequencies with those from the parked condition, it seems reasonable to ascribe the peak near $0.27 \mathrm{~Hz}$ to the tower bending modes and the peak near $0.95 \mathrm{~Hz}$ to the edgewise blade modes. The parked turbine did not have any modes between 0.9 and $1.5 \mathrm{~Hz}$, but the peak in the tower spectrum at $1.15 \mathrm{~Hz}$ can be understood to be a forward whirling mode due to the two edgewise blade modes at $0.95 \mathrm{~Hz}\left(0.95 \mathrm{~Hz}+\omega_{A}\right)$. One would also expect to see the other of the two edgewise modes turn into a backward whirling mode as the blade rotates and appear at $0.95 \mathrm{~Hz}-\omega_{A}$. There is a very weak peak near $0.75 \mathrm{~Hz}$ so this is probably the case.

There is one coherent peak in the PSD that is not explained by the linear time invariant system, the peak occurring at $1.35 \mathrm{~Hz}$ in the blade responses. In the blade's reference frame the edgewise modes should not shift in frequency due to the rotation of the propeller, and the fact that this peak is spaced from the $0.95 \mathrm{~Hz}$ modes by an integer multiple of the rotation frequency suggests that it might be due to time-periodic effects. This warrants further investigation using the output-only identification presented in Section 2. Before doing so the following filtering was performed to attempt to minimize the noise in the signal due to the harmonics of the rotation frequency. First, the simulated measurement was resampled synchronous with the best-fit rotation frequency, which was found to be $0.20167 \mathrm{~Hz}$. Then the FFT was found and all of the harmonics of the rotation frequency 
were deleted from the FFT. The inverse FFT of this signal was then found and used in the subsequent processing. This is potentially a non-causal filtering process, but the harmonics were troublesome so such a drastic approach seemed justified.

The edgewise response of blade 3 at the tip was expanded using eq. (8) with $n=-2 \ldots 2$ (so $\mathbf{y}$ was a 5 by 1 vector), and the power spectrum $\mathrm{S}_{\mathrm{yy}}$ was found using eq. (11). The primary row in that matrix is shown in Figure 5 from 0 to $1.6 \mathrm{~Hz}$. The largest peak in the spectrum occurs in the $(0,0)$ term in the PSD matrix near $0.95 \mathrm{~Hz}$, suggesting that the constant term in that mode's time-periodic mode shape is dominant. A coherent peak also occurs in the $(0,2)$ element of the PSD at that same frequency, suggesting that a second harmonic is also important to that mode. The peak picking method was used to estimate the Fourier coefficients for each mode at the $0.95,1.15$ and $1.35 \mathrm{~Hz}$ peaks. Each Fourier coefficient was normalized by the maximum coefficient, which was ascribed to $\bar{C}_{r, 0}$, and the normalized coefficients are shown in Table 2. The five coefficients estimated at each frequency are offset in the table so that the rows correspond to $\bar{C}_{r, n}$ for a certain $n$. All three columns consistently give the real part of the $n=2$ coefficient as about $5 \%$ of the fundamental, with the imaginary part being a consistent proportion of the real part. The second column $(1.15 \mathrm{~Hz})$ differs from the other two in the estimation of the $n=1$ coefficient, but visual inspection of the data from which that coefficient was derived (the red $(0,0)$ curve in Figure 5) suggests that the true coefficient is buried in the response of the fundamental, so it should probably not be trusted. The $1^{\text {st }}$ and $3^{\text {rd }}$ columns consistently give that Fourier coefficient as less than $2 \%$ of the fundamental.

The coefficients for $n<0$ are more problematic. The spectra that these coefficients are derived from are very noisy, due to the harmonic response of the turbine that was discussed previously. For example, the $(0,-2)$ curve would yield the $n=-2$ coefficient near $0.95 \mathrm{~Hz}$, but that peak is very noisy, so the coefficient doesn't seem trustworthy. The peaks below $0.95 \mathrm{~Hz}$ are incoherent due to the noise, so they cannot be used either. It seems that an improved method must be devised for filtering out the harmonic contributions before the coefficients for $n<0$ can be confidently estimated. Nevertheless, the peak at $0.95 \mathrm{~Hz}$ suggests that the $n=-1$ and $n=-2$ coefficients are less than $2 \%$ of the fundamental, so if that information is reliable, then perhaps they can be neglected.

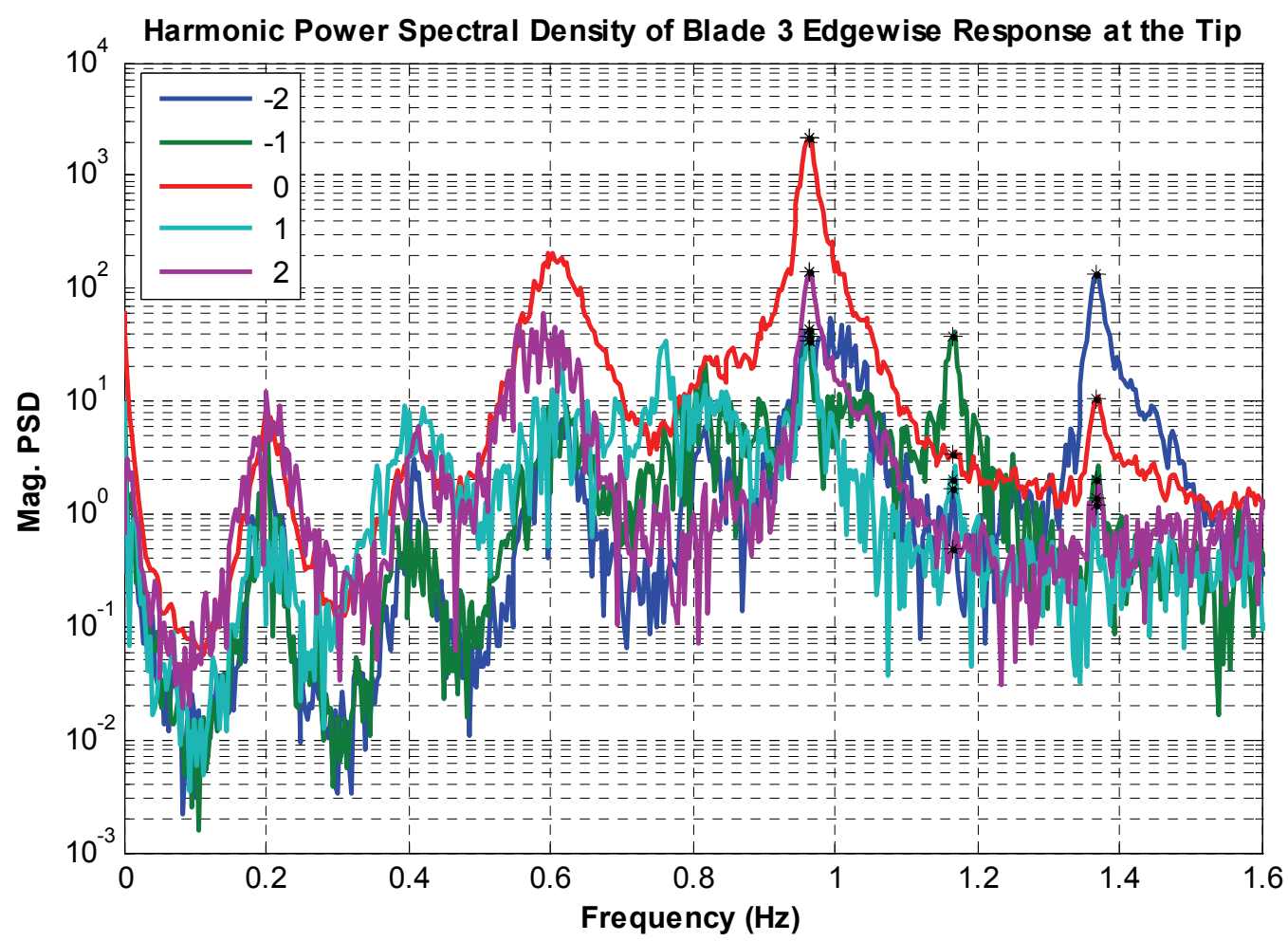

Figure 5: Harmonic PSD of the edgewise response of the tip of blade 3, computed using eqs. (8) and (11). Five curves are shown corresponding to the $m$, nth elements of $S_{y y}$ for $m=0$ and $n=-2 \ldots 2$. 


\begin{tabular}{c|ccc} 
& \multicolumn{4}{|c}{ Fourier Coefficients of B3-Tip EW Mode Shapes: $\bar{C}_{r, n}$} \\
& $\mathbf{0 . 9 5 ~ H z}$ & $\mathbf{1 . 1 5} \mathbf{~ H z}$ & $\mathbf{1 . 3 5} \mathbf{~ H z}$ \\
\hline $\mathbf{- 2}$ & $-0.0013+0.020 i$ & - & - \\
$\mathbf{- 1}$ & $-0.00052+0.015 i$ & $0.011+0.043 i$ & - \\
$\mathbf{0}$ & 1 & 1 & 1 \\
$\mathbf{1}$ & $0.017+0.0041 i$ & $0.090+0.021 i$ & $0.015+0.0014 i$ \\
$\mathbf{2}$ & $-0.053-0.033 i$ & $-0.043-0.035 i$ & $-0.066-0.041 i$ \\
$\mathbf{3}$ & - & $-0.0028-0.013 i$ & $0.0068+0.0056 i$ \\
$\mathbf{4}$ & - & - & $0.0039+0.0095 i$
\end{tabular}

Table 2: Normalized Fourier Coefficients for the mode shape at the tip of blade 3 in the edgewise direction. Each column gives an estimate of the same Fourier coefficients.

\subsection{Discussion}

In [55] it was noted that the turbulence box used in the wind turbine model had a finite length and was repeated several times during the course of the simulation, so for the parked turbine sharp harmonic content was observed at $1.354 \mathrm{~Hz}$ and its multiples. It is possible that the peak in the blade response spectra at 1.35 in Figure 4 might also be due to this effect, but there is considerable evidence to the contrary. First, the turbine is rotating at $\omega_{A}=0.20157 \mathrm{~Hz}$, and $1.354 \mathrm{~Hz}$ is not evenly divisible by $0.20157 \mathrm{~Hz}$, so each time the turbulence box repeats the blades are in a different orientation causing them to see a non-periodic pattern of turbulence. Second, the measurements from the rotating turbine do not show peaks at $2.708 \mathrm{~Hz}, 4.062 \mathrm{~Hz}$ or any other integer multiples of $1.354 \mathrm{~Hz}$, further confirming that the turbine's response is not periodic due to the repeating turbulence box. Third, the shape of the peak seen in Figure 4 at $1.35 \mathrm{~Hz}$ is not at all like the peaks due to the repeating turbulence box in the parked measurements [55]. Those peaks were sharp, nearly single-line peaks and the spectra around them had a strange character as well. Finally, when the LTP identification method is applied to the turbine responses, the spectra of the exponentially modulated response in Figure 5 show numerous peaks at integer multiples of $0.20157 \mathrm{~Hz}$. This means that the turbine's response at distinct frequencies that are separated by $0.20157 \mathrm{~Hz}$ is coherent, evidence that the system is time periodic. If the response at $\left(\omega \pm \omega_{A}\right)$ were not coherent then the spectra would be weak and have the appearance of noise at these frequencies. For example, notice the peak in the $(0,-2)$ element of the PSD matrix (blue). At $0.95 \mathrm{~Hz}$ that element of the PSD matrix is the product of the response at 0.95 $\mathrm{Hz}$ and that at $0.95-2 * 0.20157=0.55 \mathrm{~Hz}$. Figure 4 shows that the response at $0.55 \mathrm{~Hz}$ is large due to bleeding of the harmonics of the rotational frequency, yet this peak does not particularly stand out in the $(0,-2)$ element of the PSD, presumably because the response at $0.55 \mathrm{~Hz}$ is not systematically related to that at $0.95 \mathrm{~Hz}$.

\section{Conclusions}

A new output-only system identification routine was proposed that uses only the output spectra to estimate the modal parameters of a linear time-periodic system up to a scale factor. The proposed method makes used of the theory developed by Wereley regarding Harmonic Transfer Functions, and an analytical expression for the output spectrum in terms of the modal parameters was developed and used to interpret the spectra. The method was validated using simulated measurements from a Mathieu Oscillator, illustrating the simplicity of the approach and the similarity with familiar output-only identification approaches for linear timeinvariant systems. The methodology was also applied to simulated measurements from a large, detailed model of a rotating wind turbine. The results suggest that the first edgewise modes of the turbine were measurably timeperiodic. The dominant term in the Fourier expansion of the mode shape for the mode in question was the second term, which seems reasonable considering that the boundary condition imparted by the tower changes its effective stiffness twice per revolution of the rotor (in the blade's reference frame). Further results were not yet available at the time this article was submitted to the conference, but future works will explore these issues in more detail and seek to better understand why this time-periodicity occurs and how it might affect the stability and fatigue life of the wind turbine. 


\section{References}

[1] J. Dugundji and J. H. Wendell, "SOME ANALYSIS METHODS FOR ROTATING SYSTEMS WITH PERIODIC COEFFICIENTS," AIAA journal, vol. 21, pp. 890-897, 1983.

[2] C. Fuehne, "Application of Generalized Floquet Theory to Ground Resonance Data," in 56th Annual National Forum of the American Helicopter Society Virginia Beach, VA, 2000.

[3] S. Hwang, "Frequency Domain System Identification of Helicopter Rotor Dynamics Incorporating Models with Time Periodic Coefficients," in Department of Aerospace Engineering. vol. Doctor of Philosophy: University of Maryland - College Park, 1997.

[4] D. A. Peters, "Fast Floquet theory and trim for multi-bladed rotorcraft," Journal of the American Helicopter Society, vol. 39, pp. 82-89, 1994.

[5] A. Siddiqui, "Identification of the Harmonic Transfer Functions of a Helicopter Rotor," in Department of Aeronautics and Astronautics. vol. M.S.: Massachusetts Institute of Technology, 1999, p. 88.

[6] V. Verdult, M. Lovera, and M. Verhaegen, "Identification of linear parameter-varying state-space models with application to helicopter rotor dynamics," International Journal of Control, vol. 77, pp. 1149 - 1159 , 2004.

[7] C. P. Fuehne, "Application of Generalized Floquet Theory to Numerical and Experimental Data," in Mechanical Engineering. vol. M.S. St. Louis, Missouri: Washington University in St. Louis,, 2000.

[8] I. Bucher and O. Shomer, "Detecting asymmetry in rotating structures a combined acutation and signal processing approach," in 23rd International Modal Analysis Conference (IMAC XXIII) Orlando, Florida, 2005.

[9] M. Allen and J. H. Ginsberg, "Floquet Modal Analysis to Detect Cracks in a Rotating Shaft on Anisotropic Supports," in 24th International Modal Analysis Conference (IMAC XXIV) St. Louis, MO, 2006.

[10] M. S. Allen, "Frequency-Domain Identification of Linear Time-Periodic Systems using LTI Techniques," Journal of Computational and Nonlinear Dynamics vol. 4, 24 Aug. 20092009.

[11] G. Bir and K. Stol, "Operating Modes of a Teetered-Rotor Wind Turbine," in 17th International Modal Analysis Conference (IMAC XVII) Kissimmee, Florida, 1999.

[12] D. J. Malcolm, "Modal response of 3-bladed wind turbines," Journal of Solar Energy Engineering, Transactions of the ASME, vol. 124, pp. 372-377, 2002.

[13] K. Stol, M. Balas, and G. Bir, "Floquet modal analysis of a teetered-rotor wind turbine," Journal of Solar Energy Engineering-Transactions of the ASME, vol. 124, pp. 364-371, Nov 2002.

[14] J. B. Dingwell and J. P. Cusumano, "Nonlinear time series analysis of normal and pathological human walking," Chaos, vol. 10, pp. 848-63, 2000.

[15] J. B. Dingwell and K. Hyun Gu, "Differences between local and orbital dynamic stability during human walking," Transactions of the ASME. Journal of Biomechanical Engineering, vol. 129, pp. 586-93, 2007.

[16] Y. Hurmuzlu and C. Basdogan, "On the measurement of dynamic stability of human locomotion," Journal of Biomechanical Engineering, Transactions of the ASME, vol. 116, pp. 30-36, 1994.

[17] Y. Hurmuzlu, C. Basdogan, and D. Stoianovici, "Kinematics and dynamic stability of the locomotion of post-polio patients," Transactions of the ASME. Journal of Biomechanical Engineering, vol. 118, pp. 40511, 1996.

[18] M. S. Allen and M. W. Sracic, "System Identification of Dynamic Systems with Cubic Nonlinearities Using Linear Time-Periodic Approximations," in ASME 2009 International Design Engineering Technical Conferences \& Computers and Information in Engineering Conference IDETC/CIE 2009 San Diego, California, USA, 2009.

[19] S. Maia, J. M. M. Silva, J. He, N. A. Lieven, R. M. Lin, G. W. Skingle, W. M. To, and A. P. V. Urgueira, Theoretical and Experimental Modal Analysis. Taunto, Somerset, England: Research Studies Press Ltd., 1997.

[20] K. Liu, "Identification of linear time-varying systems," Journal of Sound and Vibration, vol. 206, pp. 487505, 1997.

[21] M. Verhaegen and Y. Xiaode, "A class of subspace model identification algorithms to identify periodically and arbitrarily time-varying systems," Automatica, vol. 31, pp. 201-16, 1995.

[22] F. Felici, J. W. van Wingerden, and M. Verhaegen, "Subspace identification of MIMO LPV systems using a periodic scheduling sequence," Automatica, vol. 43, pp. 1684-97, 2007.

[23] J. J. Hench, "A technique for the identification of linear periodic state-space models," International Journal of Control, vol. 62, pp. 289-301, 1995.

[24] D. A. Peters and X. Wang, "Generalized Floquet Theory for Analysis of Numerical or Experimental Rotor Response Data," in 24th European Rotorcraft Forum Marseilles, France, 1998. 
[25] O. A. Bauchau and J. Wang, "Efficient and robust approaches to the stability analysis of large multibody systems," Journal of Computational and Nonlinear Dynamics, vol. 3, 2008.

[26] P. O. Arambel and G. Tadmor, "Robust H infinity identification of linear periodic discrete-time systems," International Journal of Robust and Nonlinear Control, vol. 4, pp. 595-612, 1994.

[27] V. Deshmukh, "Spectral Collocation-Based Optimization in Parameter Estimation of Nonlinear TimeVarying Dynamical Systems " in ASME 2007 International Design Engineering Technical Conference. vol. DETC2007-35262 Las Vegas, Nevada, 2007.

[28] N. M. Wereley, "Analysis and Control of Linear Periodically Time Varying Systems," in Department of Aeronautics and Astronautics. vol. PhD Cambridge: Massachusetts Institute of Technology, 1991.

[29] N. M. Wereley and S. R. Hall, "Linear time periodic systems: transfer functions, poles, transmission zeroes and directional properties," in Proceedings of the 1991 American Control Conference Boston, MA, USA: American Autom. Control Council, 1991, pp. 1179-84.

[30] N. M. Wereley and S. R. Hall, "Frequency response of linear time periodic systems," Honolulu, HI, USA, 1990, pp. 3650-3655.

[31] H. Irretier, "MATHEMATICAL FOUNDATIONS OF EXPERIMENTAL MODAL ANALYSIS IN ROTOR DYNAMICS," Mechanical Systems and Signal Processing, vol. 13, pp. 183-191, 1999.

[32] C.-W. Lee, D.-J. Han, J.-H. Suh, and S.-W. Hong, "Modal analysis of periodically time-varying linear rotor systems," Journal of Sound and Vibration, vol. 303, pp. 553-574, 2007.

[33] S. J. Shin, C. E. S. Cesnik, and S. R. Hall, "System identification technique for active helicopter rotors," Journal of Intelligent Material Systems and Structures, vol. 16, pp. 1025-38, 2005.

[34] M. S. Allen and M. W. Sracic, "A Method for Generating Pseudo Single-Point FRFs from Continuous Scan Laser Vibrometer Measurements," in 26th International Modal Analysis Conference (IMAC XXVI) Orlando, Florida, 2008.

[35] M. S. Allen and M. W. Sracic, "Mass Normalized Mode Shapes Using Impact Excitation and ContinuousScan Laser Doppler Vibrometry," in 8th International Conference on Vibration Measurements by Laser Techniques Ancona, Italy, 2008, pp. 7098-3.

[36] M. S. Allen and M. W. Sracic, "A New Method for Processing Impact Excited Continuous-Scan Laser Doppler Vibrometer Measurements " Mechanical Systems and Signal Processing, vol. submitted Jan. 2009, 2009.

[37] M. Martarelli, "Exploiting the Laser Scanning Facility for Vibration Measurements," in Imperial College of Science, Technology \& Medicine. vol. Ph.D. London: Imperial College, 2001.

[38] R. Ribichini, D. Di Maio, A. B. Stanbridge, and D. J. Ewins, "Impact Testing With a Continuously-Scanning LDV," in 26th International Modal Analysis Conference (IMAC XXVI) Orlando, Florida, 2008.

[39] P. Sriram, S. Hanagud, and J. I. Craig, "Mode shape measurement using a scanning laser doppler vibrometer," International Journal of Analytical and Experimental Modal Analysis, vol. 7, pp. 169-178, 1992.

[40] A. B. Stanbridge and D. J. Ewins, "Modal testing using a scanning laser Doppler vibrometer," Mechanical Systems and Signal Processing, vol. 13, pp. 255-70, 1999.

[41] A. B. Stanbridge, M. Martarelli, and D. J. Ewins, "Measuring area vibration mode shapes with a continuous-scan LDV," Measurement, vol. 35, pp. 181-9, 2004.

[42] S. Vanlanduit, P. Guillaume, and J. Schoukens, "Broadband vibration measurements using a continuously scanning laser vibrometer," Measurement Science \&amp; Technology, vol. 13, pp. 1574-82, 2002.

[43] L. Hermans and H. Van Der Auweraer, "Modal Testing and Analysis of Structures Under Operational Conditions: Industrial Applications," Mechanical Systems and Signal Processing, vol. 13, pp. 193-216, 1999.

[44] E. Parloo, B. Cauberghe, F. Benedettini, R. Alaggio, and P. Guillaume, "Sensitivity-Based Operational Mode Shape Normalization: Application to A Bridge," in 22nd International Modal Analysis Conference (IMAC XXII) Dearborn, Michigan, 2004.

[45] B. Peeters and G. D. Roeck, "Stochastic System Identification for Operational Modal Analysis: A Review," Journal of Dynamic Systems, Measurement, and Control, vol. 123, December 2001.

[46] A. Fasana, L. Garibaldi, E. Giorcelli, and D. Sabia, "Z24 Bridge Dynamic Data Analysis by Time Domain Methods," in International Modal Analysis Conference (IMAC XIX) Kissimmee, Florida, 2001.

[47] A. Guyader and L. Mevel, "Covariance driven subspace methods : input/output vs output-only," in International Modal Analysis Conference (IMAC XXII) Dearborn, Michigan, 2004.

[48] M. Richardson and B. Schwarz, "Modal Parameter Estimation from Operating Data," in Sound and Vibration, 2003, pp. 28-36. 
[49] G. H. James and T. G. Carne, "Damping measurements on operating wind turbines using the natural excitation technique (NExT)," in 11th ASME Wind Energy Symposium presented at the Energy Sources Technology Conference and Exhibition. vol. 12 Houston, TX, USA: Publ by ASME, New York, NY, USA, 1992, pp. 75-81.

[50] P. Van Overschee and B. De Moor, Subspace Identification for Linear Systems: Theory-ImplementationApplications. Boston: Kluwer Academic Publishers, 1996.

[51] J. Jonkman, "NREL 5 MW Baseline Wind Turbine," Technical report, NREL/NWTC, 1617 Cole Boulevard; Golden, CO 80401-3393, USA 2005.

[52] M. H. Hansen, "Aeroelastic stability analysis of wind turbines using an eigenvalue approach," Wind Energy, vol. 7, pp. 133-143, 2004.

[53] M. H. Hansen, "Improved modal dynamics of wind turbines to avoid stall-induced vibrations," Wind Energy, vol. 6, pp. 179-195, 2003.

[54] M. O. L. Hansen, J. N. Sørensen, S. Voutsinas, N. Sørensen, and H. A. Madsen, "State of the art in wind turbine aerodynamics and aeroelasticity," Progress in Aerospace Sciences, vol. 42, pp. 285-330, 2006.

[55] S. Chauhan, M. H. Hansen, and D. Tcherniak, "Application of Operational Modal Analysis and Blind Source Separation / Independent Component Analysis Techniques to Wind Turbines," in 27th International Modal Analysis Conference (IMAC XXVII) Orlando, Florida: SPIE, 2009.

\section{Appendix A}

\subsection{Analytical Fourier Coefficients of the Floquet Mode Shapes for the Mathieu Oscillator}

The Mathieu oscillator system can be easily represented in state space according to eq. (1), with the following system matrix, $A(t)$,

$$
A(t)=\left[\begin{array}{cc}
0 & 1 \\
-\omega_{0}^{2}-\omega_{1}^{2} \cos \left(\omega_{A} t\right) & -2 \zeta \omega_{0}
\end{array}\right] .
$$

Recalling the time derivative relationship of the STM to the $A(t)$ matrix,

$$
\frac{\partial}{\partial t}\left(\Phi\left(t, t_{0}\right)\right)=A(t) \Phi\left(t, t_{0}\right)
$$

one can obtain the analytical STM by using initial conditions of $[1 ; 0]$ and $[0 ; 1]$ and integrating the equations of motion over one fundamental period. The STM at the end of the fundamental period is also known as the Floquet transition matrix, and its eigenvalues are the Floquet multipliers of the system, $\lambda_{S T M}$, which are related to the Floquet exponents $\lambda_{F l}$ by $\lambda_{S T M}=\exp \left(\lambda_{F l} * T_{A}\right)$. After extracting the Floquet exponent, $\lambda_{F l}$ the periodic Floquet mode vectors can be calculated by rearranging eq. (4) to get eq. (18),

$$
\psi_{F l}(t)=\Phi\left(t, t_{0}\right) \psi_{S T M}\left(t_{0}\right) \exp \left(-\lambda_{F l}\left(t-t_{0}\right)\right)
$$

where $\psi_{S T M}\left(t_{0}\right)$ is the eigenvector of the STM at $t=T_{A}$. Then, because the Floquet mode vectors are periodic, they can be expanded with a Fourier Series as given in eq. (5). 